\title{
Der Satz der Variationsrechnung, welcher dem Principe der kleinsten Wirkung in der Mechanik entspricht.
}

\author{
Von A. Mayer in Leipzig.
}

Wie das Princip der Erhaltung der lebendigen Kraft, so ist auch das Princip der kleinsten Wirkung nur der specielle Fall eines weit allgemeineren Satzes der Variationsrechnung. Dieser letatere Satz, der, soviel ich weiss, bisher noch nicht bemerkt worden ist, soll hier in elementarer Weise, d. h. ohne Benutzung der Hamilton'schen Theorie abgeleitet werden. Man wird aber ohne Schwierigkeit erkennen, dass derselbe im Grunde nichts Anderes ist, als eine Interpretation jener bekannten Zurückführung der Hamilton'schen partiellen Differentialgleichung für den Fall, wo dieselbe die Grundvariable nicht explicite enthält, auf eine partielle Differentialgleichung mit einer Variabeln weniger. (Vgl. hierüber Jacobi, Dynamik, 19. und 21. Vorlesung).

Es seien $y y_{1} y_{2} \ldots y_{n}$ umbekannte Functionen von $x$ und zwischen ihnen die Bedingungsgleichungen:

$$
\varphi_{1}=0, \varphi_{2}=0, \ldots . \varphi_{m}=0
$$

vorgeschrieben, deren Anzahl selbstverständlich $<n+1$ angenommen wird und in denen die Differentialquotienten der $y$ nicht vorkommen sollen. Es bezeichne ferner $f$ eine gegebene Function der Variabeln $y$ und ihrer ersten Differentialquotienten $y^{\prime}$.

Dann führt bekanntlich die Aufgabe:

„Die den $m$ Bedingungen (1) unterworfenen Functionen $y y_{1} \ldots y_{n}$ so zu bestimmen, dass die erste Variation des Integrales

gleich Null wird"

$$
V=\int_{a_{0}}^{a_{1}} f d x
$$

auf die $n+1$ Differentialgleichungen:

$$
\frac{\partial f}{\partial y_{i}}+\sum_{k=1}^{k=m} \lambda_{k} \frac{\partial \varphi_{k}}{\partial y_{i}}=\frac{d}{d x} \frac{\partial f}{\partial y_{i}^{\prime}},
$$


welche in Verbindung mit den $m$ Gleichungen (1) hinreichen, um die gesuchten Functionen $y$, sowie die Multiplicatoren $\lambda$ bis auf eine gewisse Anzahl willkürlicher Constanten zu bestimmen.

Sind überdies, natürlich unter Beachtung der Gleichungen (1), die Werthe gegeben, welche die Variabeln $y$ in den beiden gegebenen Grenzen $a_{0}$ und $a_{1}$ annehmen sollen, so lösen die durch Integration des Systems (1), (3) erhaltenen Functionen $y$ das obige System vollständig, sobald man in ihnen die willkurlichen Constanten aus diesen Grenzbedingungen bestimmt hat.

Dies vorausgeschickt, nehme ich an, dass weder die Function $f$, noch auch die Functionen $\varphi$ die unabhängige Variable $x$ selbst enthalten.

Dann ist stets:

$$
y^{\prime} \frac{\partial f}{\partial y^{\prime}}+y_{1}^{\prime} \frac{\partial f}{\partial y_{1}^{\prime}}+\cdots y_{n}^{\prime} \frac{\partial f}{\partial y_{n}^{\prime}}-f=h
$$

ein Integral der Gleichungen (1), (3). In der That sieht man leicht, dass der Differentialquotient dieses Ausdruckes identisch verschwindet durch die Gleichungen (3) und durch diejenigen Gleichungen, die man aus (1) durch Differentiation erhält.

Die Variable $x$ kommt auch in der Gleichung (4) nicht vor. Führt man daher $y$ an Stelle von $x$ als unabhängige Variable ein, indem man allgemein setzt:

$$
y_{i}^{\prime}=\frac{d y_{i}}{d y} \cdot y^{\prime}
$$

so wird die Gleichung (4), nach $y^{\prime}$ aufgelöst, für diese Grösse einen Werth von der Form liefern:

worin $w$ eine Function ist von

$$
y^{\prime}=w \text {, }
$$

$$
y \quad y_{1} y_{2} \ldots y_{n} \frac{d y_{1}}{d y} \frac{d y_{2}}{d y} \ldots \frac{d y_{n}}{d y}
$$

Indem man diesen Werth von $y^{\prime}$, sowie die aus ihm folgenden Werthe von:

$$
y_{i}^{\prime}=\frac{d y_{i}}{d y} \cdot w
$$

in $f$ einsetzt, wird dieses ebenfalls eine Function jener Grössen, und wenn man diese Function mit $F$ bezeichnet, so hat man durch (5) identisch:

$$
f=F \text {. }
$$

Durch partielle Differentiation nach $y_{i}$ und $\frac{d y_{i}}{d y}$ entsteht hieraus:

$$
\begin{aligned}
& \frac{\partial F}{\partial y_{i}}=\frac{\partial f}{\partial y_{i}}+\frac{\partial f}{\partial y^{\prime}} \frac{\partial y^{\prime}}{\partial y_{i}}+\frac{\partial f}{\partial y_{i}^{\prime}} \frac{\partial y_{1}^{\prime}}{\partial y_{i}}+\cdots \frac{\partial f}{\partial y_{n}^{\prime}} \frac{\partial y_{n}^{\prime}}{\partial y_{i}} \\
& \frac{\partial F}{\partial \frac{d y_{i}}{d y}}=\quad \frac{\partial f}{\partial y^{\prime}} \frac{\partial y^{\prime}}{\partial \frac{d y_{i}}{d y}}+\frac{\partial f}{\partial y_{i}^{\prime}} \frac{\partial y_{1}^{\prime}}{\partial \frac{y_{i}}{d y}}+\cdots \frac{\partial f}{\partial y_{n}^{\prime}} \frac{\partial y_{n}^{\prime}}{\partial \frac{d y_{i}}{d y}} \text {. }
\end{aligned}
$$


Nach (5) ist aber:

$$
\begin{aligned}
& \frac{\partial y_{k}^{\prime}}{\partial y_{i}}=\frac{d y_{k}}{d y} \quad \frac{\partial w}{\partial y_{i}} \\
& \frac{\partial y_{k}^{\prime}}{\partial \frac{d y_{i}}{d y}}=\frac{d y_{k}}{d y} \quad \frac{\partial w}{\partial \frac{d y_{i}}{d y}} \quad \text { für } k \geqq i \text { und: } \\
& \frac{\partial y_{i}^{\prime}}{\partial \frac{d y_{i}}{d y}}=\frac{d y_{i}}{d y} \quad \frac{\partial w}{\partial \frac{d y_{i}}{d y}}+w .
\end{aligned}
$$

Führt man diese Werthe in die obigen Formeln ein und macht von der aus (4), (5) und (6) folgenden Relation

$$
w\left\{\frac{\partial f}{\partial y^{\prime}}+\frac{\partial f}{\partial y_{1}^{\prime}}, \frac{d y_{1}}{d y}+\cdots \frac{\partial f}{\partial y_{n}^{\prime}} \frac{d y_{n}}{d y}\right\}=F+h
$$

Gebrauch, so ergiebt sich:

$$
\begin{aligned}
& \frac{\partial F}{\partial y_{i}}=\frac{\partial f}{\partial y_{i}}+\frac{F+h}{w} \frac{\partial w}{\partial y_{i}} \\
& \frac{\partial F}{\partial \frac{d y_{i}}{d y}}=w \frac{\partial f}{\partial y_{i}^{\prime}}+\frac{F+h}{w} \frac{\partial w}{\partial \frac{d y_{i}}{d y}}
\end{aligned}
$$

und hieraus erhält man:

$$
\begin{aligned}
\frac{\partial f}{\partial y_{i}} & =w \frac{\frac{F+h}{w}}{\partial y_{i}} \\
\frac{\partial f}{\partial y_{i}^{\prime}} & =\frac{\frac{F+h}{w}}{\partial \frac{y_{i}}{d y}} .
\end{aligned}
$$

Substituirt man endlich diese Werthe in die $n$ letzten Gleichungen (3), dividirt durch $w$ und setzt:

$$
\begin{aligned}
d x & =\frac{1}{w} d y, \\
\frac{\lambda_{k}}{w} & =\mu_{k},
\end{aligned}
$$

so gehen diese Gleichungen über in:

$$
\frac{\partial \frac{F+h}{w}}{\partial y_{i}}+\sum_{k=1}^{k=n t} \mu_{k} \frac{\partial \varphi_{k}}{\partial y_{i}}=\frac{d}{d y} \frac{\partial \frac{F+h}{w}}{\partial \frac{d y_{i}}{d y}} .
$$

Die Lösungen des Systems (1), (3) erfüllen mithin, nachdem man in ihnen vermittelst des Werthes von $y$ diese Variable an Stelle von $x$ als unabhängige Variable eingeführt hat, anch die $n$ Gleichungen (8) identisch. 
Diese Gleichungen sind aber nichts Anderes als die Differentialgleichungen des Problems:

„Unter der Voranssetzung, dass stets die Bedinymngen (1) erfült sein sollen, diejenigen Functionen $y_{1} y_{2} \ldots y_{n}$ zu finden, fiir welche die erste Variation des Integrales

versehwindet."

$$
W=\int_{b_{0}}^{b} \frac{F+h}{w} d y
$$

Schreibt man daher in diesem zweiten Integrale den Grenzen $b_{*}$ und $b_{1}$ von $y$, sowie den Grenzwerthen von $y_{1} y_{2} \ldots y_{n}$ dieselben Werthe vor, die diese Variabeln in der ersten Aufgabe für $x=a_{0}$ und $x=a_{1}$ annehmen sollten, und giebt der Constanten $h$ den Werth, den sie dort erhält, so bringen die Lösungen des ersten Problems gleichzeitig und unter denselben Beschrïnkungen (1) auch die erste Variation des Integrales (9) zum Verschwinden.

Das Integral (9) aber entsteht aus dem Integrale:

$$
\int_{\omega_{0}}^{u_{1}^{\prime}} d x\left(y^{\prime} \frac{\partial f}{\partial y^{\prime}}+y_{i}^{\prime} \partial y_{i}^{\prime}+\cdots y_{n}^{\prime} \partial y_{n}^{\prime}\right)
$$

wern man die unabhängige Variable $x$ mittelst der Gleichung (4) eliminirt.

Hat man umgekehrt die allgemeinen Gleichungen $(1),(8)$ des zweiten Problems vollständig integrirt, so braucht man, um die vollständigen Lösungen der Gleichungen des ersten Problems z.u erhalten, nur noch die eine Quadratur:

auszuführen. -

$$
x+\text { const. }=\int \frac{11}{w} d y
$$

Dass dieser Satz in der That das Princip der kleinsten Wirkung als speciellen Fall enthält, zeigt sich sofort, wenn man

$$
x=t, f=T+U
$$

setzt, wo $U$ eine Function der Coordinaten $x_{1} y_{1} z_{1}, x_{2} y_{2} z_{2}, \ldots$, die jetzt an die Stelle der unbekannten Functionen $y$ treten, md

$$
T=\frac{1}{2} \underset{i}{\Sigma} m_{i}{ }^{\prime}\left(x_{i}^{\prime 2}+y_{i}^{\prime 2}+z_{i}^{\prime 2}\right)
$$

ist. Denn hierdurch verwandelt sich das Integral (2) in das Ha milton'sche Integral

$$
\int(T+U) d t
$$

die Gleichung (4) in den Satz der lebendigen Kraft

$$
T=U+h
$$

und das Integral (10) in das Integral des Princips der kleinsten Wirkung 


$$
\int d t \cdot 2 T=\int \sum_{i} m_{i} v_{i} d s_{i}
$$

Der obige Satz ist übrigens auch einer Ausdehnung auf den allgemeinen Fall der Variationsrechnung fähig, d. h. man kann entsprechende Sätze auch für den Fall aufstellen, wo die Bedingungsgleichungen (1) die Differentialquotienten $y^{\prime}$ enthalten. Nur, weil in das Integral, welches alsdann an die Stelle der Gleichung (4) tritt, auch die Multiplicatoren $\lambda$ eingehen, scheint hier nichts zu existiren, was dem Principe der kleinsten Wirkung vollkommen analog wäre. Wichtiger aber als diese directe Verallgemeinerung dürfte ein damit nahe verwandter Satz sein, der Satz nämlich, dass man im Allgemeinen immer eine der Bedingungsdifferentialgleichungen ganz eliminiren kann, sobald weder in dem Integrale, noch in den Bedingungsgleichungen die nuabhängige Variable explicite auftritt.

In der That, die Differentialgleichungen des Problems

$$
\delta \int f d x=0
$$

zu machen, während zwischen den unbekannten Functionen $y$ die Differentialgleichung erster Ordnung

besteht, sind:

$$
\varphi=0
$$

$$
\frac{\partial \Omega}{\partial y_{i}}=\frac{d}{d x} \frac{\partial \Omega}{\partial y_{i}^{\prime}},
$$

worin $\Omega=f+\lambda \varphi$ ist, und wenn weder $f$ noch $\varphi$ die Variable $x$ selbst enthält, so gilt das Integral:

$$
\sum_{i} \frac{\partial \Omega}{\partial y_{i}^{\prime}} y_{i}^{\prime}-f=h \text {. }
$$

Unter dieser Annahme und vorausgesetzt, dass $\varphi$ nicht gerade homogen in Bezug auf die Differentialquotienten $y^{\prime}$ ist, kann man aber, wenn man wieder

$$
y_{i}^{\prime}=\frac{d y_{i}}{d y} y^{\prime}
$$

setzt, aus der Gleichung $\varphi=0 y^{\prime}$ ausdrücken durch

$$
y y_{1} \ldots y_{n}, \frac{d y_{1}}{d y} \cdots \frac{d y_{n}}{d y},
$$

und wenn man diesen Werth mit $\omega$ und mit $F$ denjenigen Ausdruck bezeichnet, in den $f$ durch die Substitutionen:

$$
y_{i}^{\prime}=\frac{d y_{i}}{d y} \cdot \omega
$$

übergeht, so hat man durch diese Substitutionen identisch:

$$
\Omega=F \text {. }
$$

Indem man diese Identität nach $y_{i}$ und $\frac{d y_{i}}{d y}$ differentiirt, das In- 
A. Mathr.

tegral (12) anwendet und mit Hülfe der anf diese Weise erhaltenen Werthe von

$$
\frac{\partial \Omega}{\partial y_{i}} \quad \text { und } \quad \frac{\partial \Omega}{\partial y_{i}^{\prime}}
$$

die Differentialgleichungen (11) umformt, sieht man auf ganz demselben Wege wie vorhin, dass die Lösungen der Differentialgleichungen des betrachteten Problems gleichzeitig auch die $n$ Differentialgleichungen lösen:

$$
\frac{\partial \frac{F+h}{\omega}}{\partial y_{i}}=\frac{d}{d y} \frac{\partial \frac{F+h}{\partial \frac{\omega}{d y_{i}}}}{d y} .
$$

Diese Gleichangen sind aber die Differenzialgleichungen des unbeschränkten Problems: diejenigen Functionen $y_{1} y_{2} \ldots y_{n}$ zu finden, welche die erste Variation des Integrales

$$
\int \frac{F+h}{\omega} d y
$$

zum Verschwinden bringen, und dieses Integral erhält man aus dem Integrale

$$
\int \sum_{i} \frac{\partial \Omega}{\partial y_{i}^{\prime}} y_{i}^{\prime} d x
$$

indem man $x$ mittelst der Bedingungsgleichung $\varphi=0$ und $\lambda$ mittelst des Integrales (12) eliminirt.

Wären ausser der Bedingung $\varphi=0$ noch andere Bedingungen $\varphi_{1}=0, \varphi_{2}=0, \ldots$ gegeben, entweder zwischen den Functionen $y$ allein, oder zwischen diesen und ihren ersten Differentialquotienten, jedoch immer ohne $x$, so hätte man in dem Vorhergehenden nur überall $f+\lambda_{1} \varphi_{1}+\lambda_{2} \varphi_{2}+\cdots$ statt $f$ zu setzen.

Wendet man dies Resultat beispielsweise auf die Aufgabe an: $x, y, z$ als Functionen von $s$ so zu bestimmen, dass

$$
\delta \int f(x, y, z) d s=0
$$

werde, während stets:

$$
\left(\frac{d x}{d s}\right)^{2}+\left(\frac{d y}{d s}\right)^{2}+\left(\frac{d z}{d s}\right)^{2}=1
$$

sein soll, so erhält man mit Rücksicht auf die statische Bedeutung dieser Aufgabe den Satz:

Die Gleichgewichtslage eines unausdehnbaren Fadens, der an seinen beiden Enden befestigt ist und von Kräften gespannt wird, die eine Kräftefunction $f(x, y, z)=U$ besitzen, bestimmt sich aus der Bedingung:

$$
\delta \int(U+h) \sqrt{1+\left(\frac{d y}{d x}\right)^{2}+\left(\frac{d z}{d x}\right)^{2}} d x=0
$$


und dies ist der wahre Ausdruck des Princips, welches Möbius in $\S 305$ seines Lehrbuchs der Statik als Analogon des Princips der kleinsten Wirkung für das Fadengleichgewicht aufstellt und dort so ausspricht, dass das Integral $\int T d s$ für die Fadencurve ein Maximum oder Minimum sei. Es ist aber hinzuzufügen, dass man die Spannung $T$ durch dasjenige Integral der statischen Gleichungen, welches dem Satze der lebendigen Kraft in der Dynamik entspricht, und $d s$ durch die Bedingung der Unausdehnbarkeit des Fadens eliminiren muss. 